\title{
Improving phlebotomy handover of untaken blood tests to junior doctors
}

Emily Brown, Helen Jarrett, Gabrielle Churchhouse, Matthew Hartley, David Cohen, Katerina Kyprianou, Sarah Murphy, Brindley Walker, Catherine Hill

Southmead Hospital, Bristol, UK

\begin{abstract}
A lack of communication between junior doctors and phlebotomists means untaken blood tests are often not recognised until late in a junior doctor's day, resulting in additional hours worked, delays in patient management, and potentially avoidable handover of additional work to oncall doctors.

We set out to improve communication, with an aim that ward doctors should be made aware of patients who have not been successfully bled by phlebotomists by 1:00pm. By introducing a formal handover clipboard in a designated ward space, we facilitated communication between phlebotomists and doctors, and minimised the potential for unrecognised "missed" blood tests.
\end{abstract}

Our intervention was met with approval; $88 \%$ of junior doctors surveyed stated they found the clipboards useful, and $74 \%$ have noticed an improvement in communication, working efficiency and better patient safety. Post-intervention, junior doctors knew about $70 \%$ of booked blood tests that had not been taken by $1: 00 \mathrm{pm}$, compared to $26 \%$ pre-intervention. By allowing the recognition of missed blood tests to be noted early enough in the day for repeat samples to be taken, and the results to be acted upon, we feel our intervention has been a success. As a group of new foundation doctors we have felt empowered that as a result of recognising a problem, implementing simple changes, and monitoring results we have made a genuine improvement to multi-disciplinary team working, workload of junior doctors, and patient safety.

\section{Problem}

We have identified an ongoing problem in Southmead Hospital, Bristol, UK regarding the communication between the junior doctors and phlebotomists. Patients are regularly not being bled by phlebotomists for various reasons; the patient may be away from the bedside during the phlebotomy round, or phlebotomists may struggle to take blood from patients with difficult IV access. However, junior doctors often don't find out about missed samples until late in the day when they go to check the day's blood results. This often means that there is not enough time to bleed the patients and get results back before the end of the working day. This can be dangerous for patients since it means that important treatment can be delayed. Often it also leads to longer hours worked by doctors if bloods need to be repeated and treatment initiated later in the day. Additionally, this can mean an increase in the workload handed over to the already busy on-call team in the evenings.

There is a clear need to improve phleobotomists handover to junior doctors about patients who haven't been bled that day.

Aim: Junior doctors should be made aware of patients who have not been successfully bled by phlebotomists by $1 \mathrm{pm}$.

\section{Background}

A QI project done in 2014 looked at an effective method to improve handover between phlebotomists and doctors on medical wards.[1] The intervention used was a standardised phlebotomy handover folder, where the names of patients not bled would be communicated to the doctors. The results of this project demonstrated a dramatic improvement in the communication between phlebotomists and doctors through the use of a standardised folder. Post-intervention, the proportion of bloods not taken by phlebotomists and communicated to doctors was $100 \%$.

Another QI project was done in the Evelina Children's Hospital looking at improving communication between doctors and phlebotomists in the paediatric wards.[2] Similar issues were raised where bloods not taken during the day were not communicated to the doctors on time leading to delays in discharge or treatment and junior doctor stress. Three different PDSA cycles were performed following three interventions. The interventions used involved phlebotomists bleeping the nurse in charge about bloods not taken that day. Additionally, a print out of the blood request list for the ward would be printed by the doctors and given to the nurses so that they know which patients needed to be bled that day.

Their results demonstrated that $44 \%$ of bloods not taken were communicated to the doctors post-intervention compared to $27 \%$ pre-intervention.

The results from both studies mentioned above were very encouraging and shows that good communication between the doctors and phlebotomists is achievable by simple and quick interventions.

\section{Baseline measurement}

Baseline data collected from one ward. Data were collected on the following: 
1. Number of bloods requested

2. Number of patients bled and not bled (including percentages of each)

3. Reasons for not being bled

4. Time that doctors found out about patients not being bled

5. Way that doctors found out about patients not being bled. A drop down menu was used with the following options: phlebotomists hand over sheet, verbal communication to doctors, verbal communication to ward staff, not communicated and found out when checking results

6. Consequences of not finding out about the results on time.

Results: The percentage of patients not bled at baseline measurement was $16.67 \%$. The most common way that doctors found out about the patients not being bled was while checking blood results. The average time taken for doctors to find out results from 8:00am was five hours and 36 minutes.

Junior doctors questionaire: A questionnaire was sent out to all junior doctors working in Southmead hospital in order to look at the junior doctors views regarding the communication between phlebotomists and doctors. The questionnaire contained the following questions:

1. Do you feel more could be done to improve communication between phlebotomists and junior doctors?

2. Are you confident that failed/unable blodds booked for phlebotomists will be communicated effectively to you each morning?

3. Do you often discover (when looking at results) that bloods were not taken and it is now late in the day - meaning patient care affected or working hours extended?

4. Do you currently have an identified place on your ward where you know you will find an accurate recordning of up to date phlebs blood list?

5. Would you find it useful to have such an identified place?

The responses to the questionnaire were as follows:

Ninety six percent of junior doctors felt more could be done to improve phlebotomy-doctor communication. Ninety three percent said they did not feel confident that they would have failed bloods effectively communicated to them before the intervention, with $87 \%$ not having a designated area/process for phlebotomist-doctor communication. Ninety six percent stated that late discovery of failed bloods often lead to extended working hours or a negative impact on patient care.

One hundred percent of doctors surveyed said "YES" when asked if they would find it useful to have an identified place for communication of failed bloods from the phlebotomy ward round.

\section{Design}

We were aware that no formal handover process existed between phlebotomists and doctors to pass on information about who had and hadn't been successfully bled each day, despite the huge daily importance of being able to assess patients' blood results. We identified a number of haphazard handover methods in use including phlebotomists leaving unused blood stickers in patients' medical notes or on their bedroom doors, via the phlebotomists blood sheet which may be left on the ward and may have unbled patients marked, handing the message over to the nursing stuff but the message was not always be passed on in a timely fashion, or telling a doctor on the ward who may or may not have been that particular patient's doctor. These handovers were unreliable and unpredictable and thus our aim was to introduce a standardised handover method between phlebotomists and doctors, which could be used reliably on all wards throughout the hospital.

We discussed our plans with phlebotomists and ward sisters, who agreed that a standardised handover method would be of benefit. We considered a number of options including placing unused blood stickers on the patient's bedroom door, placing unused blood stickers on the main ward board by the patient's name, placing a specific magnet (eg red star) on the main ward board by patients who had not been bled and using a central clipboard system where phlebotomists could place their daily list (with each patient ticked or crossed accorded to whether they were bled) and also attached the blood stickers for patients they had not bled. It was felt that use of a magnet had potential pitfalls in terms of patients moving beds within the ward and also required a new resource (magnet) to be created. In addition, we felt a central location was paramount for ease of access for phlebotomists and doctors. Overall, using a central clipboard system was felt to be the most beneficial.

We initially planned to attach the clipboard to the main ward board; however, during consultation with ward sisters, the important issue of patient confidentially was raised. As a result we decided that leaving the clipboard by the main ward board could breech patient confidentially. We discussed a location for the clipboard with the phlebotomists and it was decided that it should be left by the "pod" on each ward as this is an easily identifiable location. It is where the phlebotomist finish their rounds each day (to send off the bloods to the laboratory) and thus a natural place for them to update the clipboard, and it was behind the receptionist's desk so would help respect patient confidentially.

Our final decision was for a clipboard with a red laminated card on the front reading "phlebotomists", to allow easy identification of the clipboard. We would attach it to the hook by the pod on each ward. Each day at the end of their round, phlebotomists would attach their A4 blood lists to the board, identify patients they hadn't bled with a "X" and leave the unused blood stickers attached to the clipboard. The clipboards would thus be in a central place on the ward, meaning that whenever juniors doctors were passing they could look at the clipboards and quickly and easily identify which patients had not been bled and pick up the blood labels to use themselves. This intervention is cheap, sustainable, and easy to implement.

We finalised plans with the phlebotomy manager and she informed all the phlebotomists of the intervention. We planned to trial it on one ward for one week and then to implement it across the rest of the hospital over one weekend, to minimise confusion for phlebotomists as they work across a number of wards. After collecting data to assess how well this handover was being 
implemented, we informed the hospital junior doctors of the change we had made so that they could benefit from this new line of communication with the phlebotomists.

\section{Strategy}

PDSA cycle 1: We trialled the intervention on one acute medical ward for one week and collected data regarding number of bloods not completed by phlebotomists, how the ward doctors found out about these bloods, what time they found out and what affect this had. The data was positive and the plan remained to place the clipboards by the pods on each ward. We modified the clipboards with a red laminated card on the front reading 'phlebotomists', to allow easy identification of the clipboard.

PDSA cycle 2: We planned to implement the clipboards across the rest of the hospital wards over one weekend; this was to minimise confusion for phlebotomists as they work across a number of wards and this location varies day to day. The phlebotomy manager informed the phlebotomists of the intervention at one of their weekly meetings. When data collection started, it became clear that not all phlebotomists were using the clipboards. In discussion with the phlebotomy manager, it was felt this was because not all phlebotomists had been present at the meeting explaining the clipboards.

PDSA cycle 3: We had further discussion with the phlebotomy manager and, to ensure all phlebotomists were aware of the clipboards, she briefed them again at a meeting and sent out an email with the details. We collected data over two weeks on five of the hospital wards (all acute medical and surgical wards, where junior doctors involved with this project were based).

PDSA cycle 4: With our intervention established, we informed all junior doctors (via email) of our change and surveyed their experience of using the new system. We received good feedback from the doctors and phlebotomists and no further changes were made.

\section{Post-measurement}

We felt, as junior ward doctors, that handover between phlebotomists and doctors was poor and that this resulted in patients being bled late in the day, which negatively affected patient care and junior doctors working hours. Before making any intervention, we collected data from five wards to quantify the situation. Over a one week period 226 blood tests were ordered and $15 \%$ of these bloods were not taken. Junior doctors were untold about $74 \%$ of the untaken bloods. As previously described, we surveyed junior doctors to ascertain their feelings regarding phlebotomist and junior doctor communication. Of the 30 junior doctors surveyed, $96 \%$ felt more could be done to improve communication between juniors and phlebotomists, and $100 \%$ said they wanted a designated place for communication on each ward.

We introduced the clipboard handover to one ward and our preliminary data showed a positive uptake of this communication method with $100 \%$ of untaken phlebotomist bloods being handed over via the clipboard.

We then introduced the clipboards through the hospital and our data showed large scale positive effects. Over a two week period on five wards, a total of 456 blood tests were ordered for the phlebotomists. Fifteen percent of these bloods were not taken by the phlebotomists; of note, this is the same as the $15 \%$ not taken by the phlebotomists in the pre-intervention data collection phase. The ways in which doctors found out about patients who had not been bled were dramatically different before and after the clipboards were introduced. Pre-clipboard introduction $9 \%$ were found out via the phlebotomists blood sheet, $74 \%$ were untold and $17 \%$ were verbally handed over. Post-clipboard introduction $82 \%$ were found out via the clipboards, $8 \%$ were untold and $6 \%$ verbally handed over (and an unknown handover method for $4 \%$ ). Post-intervention, junior doctors knew about $70 \%$ of bloods that had not been done by 1:00pm, compared to $26 \%$ pre-intervention.

In addition, improved methods of communication via the clipboards translated into better outcomes in terms of patient care and working hours for doctors. Pre-clipboard introduction, a patient not being bled had the following affect: $12 \%$ caused a change in patient management, $21 \%$ were a safety issue, $29 \%$ caused longer hours worked by junior doctors, $4 \%$ delayed a patient's treatment and $27 \%$ lead to no change in patient management. Post-clipboard introduction, a patient not being bled had the following affect: $80 \%$ no change in management, $2 \%$ caused a change in management, $10 \%$ lead to longer hours worked by junior doctor.

After the introduction of the clipboards we sent a second questionnaire to junior doctors;

post-intervention, $88.2 \%$ of junior doctors surveyed said they had found the introduction of communication clipboards useful. Seventy seven percent stated that they now felt more confident that failed/unable bloods booked for phlebotomists will be effectively communicated each morning via the clipboards. Seventy four percent of the junior doctors stated they had noticed an improvement in communication since the introduction of the clipboards, resulting in improved working efficiency and better patient safety.

Overall the clipboard intervention has greatly improved communication between phlebotomists and doctors, enabling faster recognition of bloods that have not been performed. This in turn has produced improvements in patient care and better working hours for junior doctors.

See supplementary file: ds5752.docx - "Figure 1,2,3 - label in document"

\section{Lessons and limitations}

Our project focussed on a simple, single intervention to improve handover of missed blood collection from phlebotomists to ward staff. The hope was that this would have maximum chance of success if applied uniformly across all wards, so that all staff would 


\section{BMJ Quality Improvement Reports}

be familiar with the process, no matter which ward they work on.

Learning points:

1. We found that our approach - keeping it simple, uniform intervention, done in consultation with all stakeholders contributed greatly to the success of our project, in comparison with other strategies that had been tried on an ad-hoc basis around the hospital

2. Involving all parties (phlebotomists, ward sisters, junior doctors) affected by the change early on in the process meant they were much more supportive of our idea when we came to roll it out across the hospital

3. Simple interventions such as ours can make a dramatic difference to patient care and to doctor workload, as evidenced by the fact that $76 \%$ of doctors surveyed said they noticed an improvement on the ward in our postintervention survey

4. While our intervention showed a dramatic improvement, there is still scope for further work. For example, it would be useful to find a way to permanently secure the handover board to the ward to ensure it does not go missing - this happened on a number of occasions, despite our best efforts to secure them in one place. Fortunately, in the few wards that the board did go missing, the phlebotomists had still left their reporting sheet in the allocated place; so, while missing clipboards are an issue for the long term success of our project, this did not affect the results of our study

5. Anecdotally, several doctors reported delays to discharge prior to our project due to delayed bloods. We had several reports that this improved following our intervention; however this was not one of our outcome measures. In retrospect we feel we should have tried to quantify the reduction in discharge delay, however, as our intervention resulted in such a significant improvement for patient care, ethically it would be difficult to roll it back to get baseline data

6. There is no guarantee that the system will continue to work if the benefits of our intervention are not handed over to the next generation of junior doctors, or if the next generation of phlebotomists neglect to use the boards for whatever reason. The process needs to be entrenched in the hospital for it to stick and show long-term benefits

7. While our communications with the phlebotomists were cordial and encouraging, we can foresee situations in which staff may change and this may not be the case. To mitigate against this, it is essential that the project becomes formally endorsed by the hospital, adopted by the phlebotomy department management, and incorporated into every ward in a permanent way.

\section{Conclusion}

The failure of communication between phlebotomists and doctors, when bloods were not taken, was widely perceived to be a serious problem by junior doctors across the hospital. Our project found that this significantly increased junior doctor workload, delayed treatment, and on occasion threatened patient safety. Our project was designed to address this hospital-wide problem with a simple single intervention. We conducted pre- and post- intervention surveys to assess its impact; which was very favourable.

The project demonstrated the importance of communication between different members of the multidisciplinary team to ensure safe, efficient and timely management of patients. It's interesting to note that while the phlebotomists are considered part of the ward staff, communication with them before our project was very ad hoc and as a result less than satisfactory. Evidently, in some cases, formalising communication between different members of the MDT reaps great benefits.

\section{References}

1. Genevieve Shouls, Zakariya Jarrar, John Wickenden. Improving phlebotomy handover to doctors: a quality improvement project. BMJ Qual Improv Report 2014;3 http://qir.bmj.com/content/3/1/u204813.w2033.short?g=gtrac ker

2. Jacqueline Pitchforth, Claire Lemer. Improving the use of phlebotomy services in a paediatric inpatient setting. BMJ Qual Improv Report 2014;2 http://qir.bmj.com/content/2/2/u202230.w1115.abstract?sid= dc4a55b1-6b74-46f7-8d72-1c8b3c198442

\section{Declaration of interests}

The authors declare that they have no competing interests in regard to this project.

\section{Acknowledgements}

We would like to acknowledge the contributions of the phlebotomy team, the ward sisters and the junior doctors at Southmead Hospital, Bristol.

\section{Ethical approval}

No formal ethical approval was required for this project. 